S. L. Segal, On an identity between infinite serios of arithmetic functions R. G. Stoneham, Normal rocurring decimals, normal periodic systems, $(j, \varepsilon)$-normality, and normal numbers ........... Ян Мовер, О некоторых арифметических средних в теорик дветафункции Римана. . . . . . . . . . . . . . . . .

W. Philipp, A conjecture of Erdös on continued tractions ..... C. Pomeranoe, on composite $n$ for which $\varphi(n) \mid n-1 . \ldots$ J. B. Friedlander, on the class numbers of certain quadratic oxtensions A. Fujii, on the zeros of Dirichlot $L$-functions $(V)$. . . . . . P. Erdös, G. Jogesh Babu and K. Ramaohandra, An asymptotio formula in additive number theory. . . . . . . . . . . .

S. Chowla and. J. B. Friedlander, Some remarks on $L$-funotions and class numbers. . . . . . . . . . . . . . .

H. Lang, Uber einfache periodischo Kettenbrüche und Vermutungen von P. Chowla und s. Chowla ...............

F. S. Cater, R. B. Crittenden and C. V. Eyaden, The distribution of sequences modulo ono . . . . . . . . . . . . . . . .

H. Sarges, Eine Anwendung des Selbergschen Siebes auf algebraische Zahllkörper . . . .

E. Bombieri, Corrigendum to my paper "On twin almost primes" and

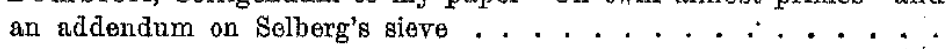

$379-380$

$387-389$

$391-393$

$395-403$

$405-4: 12$

$413-417$

$419 \sim 428$

$429-432$

$433-455$

$457-461$

La revae est consacrée à la Théorie des Nombres

The journal publishes papers on the Theory of Numbers

Die Zeitschrift veröffentlicht Arbeiten aus der Zahlentheorie

Журнал посвящен теории чпсел

L'adresse de

la Rédaction

et de l'échange

\begin{abstract}
Address of the
Editorial Board

and of the exchange

Die Adresse der

des Austausches
\end{abstract}

ACTA ARITHMETICA

al. Sniadeckiclı 8, 00-950 Warszawa

Les auteurs sont priés d'envoyer leurs manuserits en deux exemplaires The authors are requested to submit papers in two eopies

Die Autoren sind gebeten um Zusendung von 2 Exemplaren jeder Arbeit

Рукописи статей редакция просит предлагать в дпух әкземплярах

PRINTED IN POLAND

W R O C C E A W S $\mathrm{K}$ A D

\section{On an identity between infinite series of arithmetic functions}

by

S. T. SFGA (Nottingham and Rochester, N.Y.)

Let

$$
D(t)=-\frac{1}{\pi} \sum_{m=1}^{\infty} \frac{\sin 2 m \pi t}{m}=\left\{\begin{array}{lll}
\{t\}-\frac{1}{2}, & \text { if } & t \neq[t] \\
0, & \text { if } \quad t=[t]
\end{array}\right.
$$

where $\{t\}=t-[t]$.

We then obtain the formal identity

$$
\sum_{n=1}^{\infty} \frac{a_{n}}{n} D(n x)=-\frac{1}{\pi} \sum_{n=1}^{\infty} \frac{A_{n}}{n} \sin 2 n \pi x
$$

where $A_{n}=\sum_{d \mid n} a_{d}$, by substitating (1) in (2) and collecting together the terms for which mn has the same value. In general, however, this process is difficult to justify.

In [1], [2], Davenport discussed the cases where $a_{n}=\mu(n), \lambda(n)$, $\Lambda(n)$, these functions having their usual number-theoretic meanings. For rational $x$ the identity (2) is readily established using the theory of Dirichlet's $L$-series, in the sonse that, whenever one side of (2) converges, so does the other, and to the same value. Wo will refer to the identitiy (2) holding always in this sense. For irrational $x$, however, this identity appears much difficult to justify; in [1], using a reasonably complicated argument, Davenport establishes (2) in the cited cases for almost all $x$; in [2], dising Vinogradow's method which had in the meantime appeared, he establisher it for all $x$ (the proof is worked out in detail only for $a$ $=\mu(n))$. This latter proof involves the demonstration that for any fixed $h$,

$$
\sum_{n \leqslant y} \mu(n) e^{2 \pi i n x}=O\left(y(\log y)^{-h}\right)
$$

uniformly in $x$. The proof of this last is not easy, even using Vinogrador's 
The purpose of this note is to give a simple proof and natural extension of Davenport's results as to when (2) holds. It should be noted that our notation differs slightly from his, in that Davenport writes $\{t\}$ where we have $D(t)$. No contribution is made to the deeper estimates in [2]. For resultis on the Riemann $\zeta$-function $\zeta(s)$ which are used, reference may be made to [4]. $s$ and $w$ are always complex variables.

PropostrTon 1.

$$
\sum_{n=1}^{\infty} \frac{\mu(n)}{n} D(x n)=-\frac{1}{\pi} \sin 2 \pi x
$$

Proof. One of the many proofs of the functional equation for the Rjemann $\zeta$-function involves establishing the formula

$$
\zeta(s)=-s \int_{0}^{\infty} \frac{\{x\}-\frac{1}{2}}{x^{3+1}} d x=-s \int_{0}^{\infty} \frac{D(x)}{x^{s+1}} d x
$$

valid for $-1<\operatorname{Re} s<0$ (see [4], p. 15), the argument being well-known and reasonably straightforward. Making the change of variable $x=1 / u$ and applying the Mellin inversion formula gives

$$
D\left(\frac{1}{u}\right)=-\frac{1}{2 \pi i} \int_{c \rightarrow i \infty}^{c+i \infty} \frac{\zeta(s)}{s} u^{-s} d s
$$

valid for $-1<e<0$.

(The inversion may, for example, be justified by using [5], Chapter VI, Theorem (9b) with $\beta(u)=\int_{0}^{u} \frac{1}{t} D\left(\frac{1}{t}\right) d t$ and then differentiating. Alternatively (3) may be established by the argument also used below, namely, starting from the integral, apply the functional equation for the Riemann $\zeta$-function, replace $\zeta(1-s)$ by its Dirichlet series (valid since $-1<0<0$ ) interchange summation and integration, and finally use the Mellin trans form for $\sin x$ to arrive at the Fourier series (1).)

Frence we obtain

$$
\sum_{n=1}^{\infty} \frac{\mu(n)}{n} D(x n)=-\frac{1}{2 \pi i} \sum_{n=1}^{\infty} \frac{\mu(n)}{n} \int_{c-i \infty}^{c+i \infty} \frac{\zeta(s)}{s}(x n)^{s} d s
$$

for $-1<0<0$.

But $\sum_{n=1}^{\infty} \frac{\mu(n)}{n^{w}}$ converges absolutely and uniformly to $\frac{1}{\zeta(w)}$ for Rew $\geqslant 1+\varepsilon, \varepsilon>0$, and hence we may interchange the summation and integra- tion in (4), obtaining

$$
\sum_{n=1}^{\infty} \frac{\mu(n)}{n} D(x n)=-\frac{1}{2 \pi i} \int_{c-i \infty}^{c+i \infty} \frac{\zeta(s)}{s \zeta(1-s)} x^{s} d s
$$

for $-1<e<0$.

The functional equation for the Riemann $\zeta$-function and the equation $-s \Gamma(-s)=\Gamma(1-s)$ now yield

(6) $\quad-\frac{1}{2 \pi i} \int_{c-i \infty}^{a+i \infty} \frac{\zeta(s)}{s \zeta(1-s)} x^{s} d s=\frac{1}{2 \pi^{2} i} \int_{c-i \infty}^{c+i \infty} \Gamma(-s) \sin \left(\frac{1}{2} \pi s\right)(2 \pi x)^{8} d s$.

Replacing $s$ by $-s$ in $(6)$ and substituting in. (5) gives for $0<a<1$,

$$
\sum_{n=1}^{\infty} \frac{\mu(n)}{n} D(x n)=-\frac{1}{2 \pi^{2} i} \int_{a-i \infty}^{a+i \infty} \Gamma(s) \sin \left(\frac{1}{2} \pi s\right)(2 \pi x)^{-8} d s=-\frac{1}{\pi} \sin (2 \pi x),
$$

by a well-known Mellin transform (e.g. [3], p. 317, (1)).

PROPOSTTION 2.

$$
\sum_{n=1}^{\infty} \frac{\Lambda(n)}{n} D(\infty n)=-\frac{1}{\pi} \sum_{d=1}^{\infty} \frac{\log d}{d} \sin (2 \pi \infty d)
$$

Proof. Arguing exactly as in Proposition 1, since $\sum_{n=1}^{\infty} \frac{\Lambda(n)}{n^{w}}$ converges absolutely and uniformly to $\frac{-\zeta^{\prime}(w)}{\zeta(w)}$ for $\operatorname{Re} w \geqslant 1+\varepsilon, \varepsilon>0$, we obtain

$$
\begin{aligned}
\sum_{n=1}^{\infty} \frac{\Lambda(n)}{n} D(\infty n) & =\frac{-1}{2 \pi^{2} i} \int_{c-i \infty}^{c+i \infty} \Gamma(-s) \sin \left(\frac{1}{2} \pi s\right) \zeta^{\prime}(1-s)(2 \pi x)^{s} d s \\
& =\frac{1}{2 \pi^{2} i} \int_{a-i \infty}^{a+i \infty} \Gamma^{\prime}(s) \sin \left(\frac{1}{2} \pi s\right) \zeta^{\prime}(1+s)(2 \pi x)^{-s} d s,
\end{aligned}
$$

where $0<a<1$

Fere again, we may represent $-\zeta^{\prime}(w)$ by its Dirichlet series $\sum_{d=1}^{\infty} \frac{\log d}{d^{w}}$ which is absolutely and uniformly convergent for $\operatorname{Re} w \geqslant 1+\varepsilon, \varepsilon>0$, and interchange the summation and integration, thus obtaining Proposition 2 . 
Consider $\sum_{n=1}^{\infty} \frac{a_{n}}{n^{2}}$; if this series converges absolutely and uniformly for $\operatorname{Re} w \geqslant 1+\varepsilon, \varepsilon>0$, to $\mathscr{A}(w)$ say, then as is easy to see,

$$
\sum_{n=1}^{\infty} n^{-w} \sum_{d \mid r} a_{l l}
$$

converges absolutely and uniformly to $\zeta(w) . /(w)$ in the same region.

Hence, since the argument is clearly reversible, it is plain that the above argument will prove the

THEOREM. If $\sum_{n=1}^{\infty} \frac{a_{n}}{n^{w}}$ converges absolutely and uniformty for Rew $\geqslant 1+\varepsilon, \varepsilon>0$, then identity (2) holds. (In the sense that for a given w either both sides converge to the same value or both diverge.)

\section{References}

[1] H. Davenport, On some infinite series involving arithmetic functions, Quarterly Journal of Mathematics 8 (1937), pp. 8-13.

[2] - On some imfinite series involving arithmetic funotions (III), Quarturly Journal of Mathematics 8 (1937), pp. 313-320.

[3] A. Erdelyi et al., Tables of Integral Transforms I, MeGraw-Fill, 1954.

[4] E. C. Titchmarsh, Theory of Riemann Zeta-function, Oxford 1951.

[5] D.V. Widder, The Laplace Transform, Princeton 1946.

Received on 15.8 .1973

and in revised form on 6.6 .1974

\section{Normal recurring decimals, normal periodic systems, $(j, \varepsilon)$-normality, and normal numbers}

by

\author{
R. G. Stonthan (New York, N.Y.)
}

1. Introduction. In 1946, I. J. Good [1] gave a topological argument (the traversing of a particular planar network) in order to construct what he called "normal recurring decimals possessing normality of order $r$ " $\left[1\right.$, p. 167], i.e. all sequences of $r$ digits have normal frequency $10^{-r}$ in the decimal. Then he says "If $r$ is a given integer, the question arises whether there are recurring decimals possessing normality of order $r$. Any such recurring decimal (in the base 10) must clearly have a period of at least $10^{r}$. Our purpose here is to show that there are such decimals with period $10^{r}$ for any given value of $r "$. He also points out that the construction he gives can be done in any base $g$.

In 1950, Korobov [2,3 and 4, pp. 64-65] considered the normal recurring decimals of Good from at different point of view and constructed by a difforent method what he called a "normal periodic system" (still essentially a normal recurring decimal of Good) which is a positive integer $\varrho_{n}(g)$ that contains sequentially in its representation in a base $g$ all possible $n$-tuples chosen from $0,1, \ldots, g-1$. The integer $\varrho_{n}(g)$ consisting of $g^{n}+n-1$ single digits is constructed in. such a way that every n-tuple from $00 \ldots 0,00 \ldots 1, \ldots, g-1 g-1 \ldots g-1$ appears exactly once somewhere in $\varrho_{n}(g)$. For example, Korobor $[3$, p. 31] gives the normal periodic system of $2^{3}+3-1$ digits in the base $2, o_{3}(2)=1000101110$ which has each 3 -tuple, $000,001, \ldots, 111$ appearing exactly once in the sequence. Also Korobor [2] proved in 1950 , by a method different from Good's, the general existence of normal periodic systems $\varrho_{n}(g)$. Th essence, he gave an algoritihn for the construction of a normal periodic system. In [3, §4, p. 36] Korobov develops a completely general algorithm. which will produce every such $\varrho_{n}(g)$ for a given $n$ and $g$. Other papers of Korobov referenced in $[4, p p .64-65]$ studied the use of the $\varrho_{n}(g)$ in constructing a particular irrational whose distribution of fractional parts approached a uniform distribution.

The purpose of this paper is to show that the normal recurring decimals of Good and the related normal periodic systems of Korobov are very 